\title{
Metabolic differences between white and brown fat from fasting rabbits at physiological temperature
}

\author{
Z López-Ibarra', J Modrego², M Valero-Muñoz ${ }^{3}$, P Rodríguez-Sierra $^{2}$, \\ J J Zamorano-León ${ }^{2}$, A González-Cantalapiedra', N de las Heras ${ }^{2,3}$, S Ballesteros ${ }^{2,3}$, \\ V Lahera ${ }^{2,3}$ and A J López-Farré $\dot{~}^{2,4}$ \\ ${ }^{1}$ Surgery Department, Hospital Universitario ROF-Codina, Lugo, Spain \\ ${ }^{2}$ Instituto de Investigacion Sanitaria del Hospital Clínico San Carlos (IdISSC), Madrid, Spain \\ Departments of ${ }^{3}$ Physiology, and ${ }^{4}$ Medicine, School of Medicine, Universidad Complutense de Madrid, \\ Madrid 28040, Spain
}

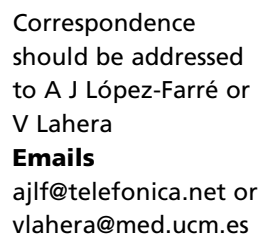

\begin{abstract}
It has been suggested that activated brown adipose tissue (BAT) shows increased glucose metabolic activity. However, less is known about metabolic activity of BAT under conditions of fasting and normal temperature. The aim of this study was to compare the possible differences in energetic metabolism between BAT and white adipose tissue (WAT) obtained from rabbits under the conditions of physiological temperature and $24 \mathrm{~h}$ after fasting conditions. The study was carried out on New Zealand rabbits $(n=10)$ maintained for a period of 8 weeks at $23 \pm 2{ }^{\circ} \mathrm{C}$. Food was removed $24 \mathrm{~h}$ before BAT and WAT were obtained. Protein expression levels of the glycolytic-related protein, glyceraldehyde-3-phosphate dehydrogenase, and pyruvate dehydrogenase were higher in WAT than that in BAT. The expression level of carnitine palmitoyltransferase 1 (CPT1) and CPT2, two fatty acid mitochondrial transporters, and the fatty acid $\beta$-oxidation-related enzyme, acyl CoA dehydrogenase, was higher in BAT than in WAT. Cytosolic malate dehydrogenase expression and malate dehydrogenase activity were higher in WAT than in BAT. However, lactate dehydrogenase expression and lactate content were significantly higher in BAT than in WAT. In summary, this study for the first time, to our knowledge, has described how under fasting and normal temperature conditions rabbit BAT seems to use anaerobic metabolism to provide energetic fuel, as opposed to WAT, where the malate-aspartate shuttle and, therefore, the gluconeogenic pathway seem to be potentiated.
\end{abstract}

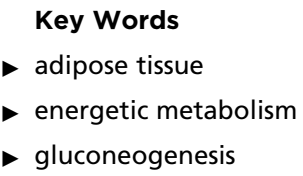

Journal of Molecular Endocrinology (2015) 54, 105-113

\section{Introduction}

Adipose tissue is well recognized as an organ designed to store excessive carbon in the form of fatty acids esterified to glycerol (triglycerides). Adipose tissue is also now recognized as the organism's main contributor to crucial survival mechanisms including the maintenance of body temperature (Lean 1989). In rodents, thermogenesis is dependent upon brown adipose tissue (BAT), while white adipose tissue (WAT) has been identified as the greatest reserve of triglycerides in the body (Dawkins \& Hull 1964).

The two main metabolic processes of WAT are lipogenesis and lipolysis. In this regard, WAT accommodates caloric excess by expanding to store triglycerides and compensates for caloric deficit through the mobilization of free fatty acids. Free fatty acids derived from WAT

Published by Bioscientifica Ltd. 
lipolysis are the principal source of lipid fuel in the fasting state. In addition to its role as fatty acid stock, WAT is needed for normal glucose homeostasis and is involved in other processes such as inflammation and various endocrine functions (Trayhurn \& Beattie 2001).

Glucose is an essential component for adipose tissue metabolism, primarily to provide glycerol-3-phosphate to form the backbone of triacylglycerol (Athenstaedt \& Daum 2006). In this regard, fasting has profound effects on in vivo glucose metabolism, because starvation results in low circulating insulin levels, promoting a state of glucose intolerance (Szepesi \& Berdanier 1971). Moreover, increased free fatty acid availability has been associated with stimulation of gluconeogenesis, involving, in organs such as the liver, regulation of key metabolic pathways such as the aspartate-malate shuttle, although the situation in the adipose tissue requires better investigation.

Unlike WAT, BAT is a mitochondria-rich tissue and expresses uncoupling protein 1 (UCP1). Sympathetic activation of BAT activates lipase-mediated triacylglycerol hydrolysis releasing fatty acids. Lipolysis-derived fatty acids are directed to the mitochondria where UCP1 promotes mitochondrial uncoupled respiration, resulting in heat generation without ATP synthesis (Lin \& Klingenberg 1980, Cannon \& Nedergaard 2004). In this regard, BAT should potentially have a marked ability to oxidize fatty acids and glucose, probably toward aerobic metabolism, due to its high mitochondrial content. Indeed, it was well established that under activated thermogenesis, BAT is a plasma-lipid-and glucose-clearing organ in rodents (Festuccia et al. 2009, Bartelt et al. 2011). In this regard, feeding with concomitantly elevated plasma insulin levels seems to increase glucose uptake by BAT (Bukowiecki 1989).

However, although several works have focused on studying the energetic metabolism in BAT under cold or diet-induced thermogenesis (Rothwell \& Stock 1983, Festuccia et al. 2009, Bartelt et al. 2011), less is known about BAT metabolism in the fasting state and the possible differences between WAT and BAT metabolism under conditions of fasting and physiological environmental temperature.

Therefore, this study aimed to compare the possible differences in energetic metabolism between BAT and WAT obtained from rabbits under the conditions of physiological temperature and $24 \mathrm{~h}$ after fasting. Specifically, this study investigated for the first time, to our knowledge, the possible differences between BAT and WAT in glucose metabolism, fatty acid $\beta$-oxidation, and the gluconeogenesis-associated aspartate-malate shuttle.

\section{Materials and methods}

\section{Experimental animals}

The study was carried out in New Zealand rabbits $(n=10$; Granja Cunicular, Navarra, Spain) weighing $2.35 \pm$ $0.023 \mathrm{~kg}$. The rabbits were maintained during 8 weeks with free access to tap water and control-fed with a standard diet (80-100 g/day; SF412 PANLAB SLU, Barcelona, Spain). Table 1 gives the composition of the diet. Rabbits were maintained under conditions of physiological temperature $\left(23 \pm 2{ }^{\circ} \mathrm{C}\right)$ with a standard $12 \mathrm{~h}$ light: $12 \mathrm{~h}$ darkness cycle. Food was removed $24 \mathrm{~h}$ before BAT and WAT were obtained.

The experimental protocol and procedures were approved by the Animal Care and Use Committee of Universidad Complutense, according to the guidelines for ethical care of experimental animals of the European Community. The rabbits were killed with an intravenous overdose $(60-65 \mathrm{mg} / \mathrm{kg}$ bw) of pentobarbitone sodium (Dolethal, Vetoquinol S.A., France) and exsanguination.

After being killed, WAT was obtained from the lumbar region. In this regard, although the different depots may have different importance in the metabolic activity of WAT, it has been reported that upper-body WAT, including that from the lumbar region, provides the major proportion of systemic nonesterified fatty acid indicating a high metabolic activity (Martin \& Jensen 1991). BAT was obtained from the interscapular depot. These fat pads are anatomically well defined and whole masses can be easily dissected. Fat pads were then weighed, washed in saline solution, dried and immediately frozen, and stored at $-80^{\circ} \mathrm{C}$ until the molecular determinations were performed.

\section{Circulating metabolic parameters}

Blood samples were collected through a catheter inserted into the ear arteries of concious rabbits. After $15 \mathrm{~min}$ of centrifugation at $1500 \mathrm{~g}$, the serum was transferred to polypropylene tubes and frozen at $-80{ }^{\circ} \mathrm{C}$ until lipids were assayed. In order to measure plasma concentrations of total cholesterol, HDL-cholesterol, and triglycerides,

Table 1 Diet composition of rabbits

\begin{tabular}{lc}
\hline & Nutritional composition (\%) \\
\cline { 1 - 1 } Proteins & 12.0 \\
Minerals ash & 6.5 \\
Moisture & 10.0 \\
Lipids & 3.0 \\
Fibers & 16.0 \\
\hline
\end{tabular}

Published by Bioscientifica Ltd. 
spectrophotometric colorimetric techniques were used, according to the manufacturer's instructions (Vitros Fusion 5,1, OrthoClinical Diagnostics, Johnson \& Johnson, New Brunswick, NJ, USA). LDL-cholesterol was also measured using an enzymatic colorimetric assay (SPINREACT, S.A./S.A.U., Gerona, Spain).

\section{Expression of energy-metabolism-related proteins}

Protein expression levels of 1,6-biphosphate aldolase, glyceraldehyde-3-phosphate dehydrogenase, pyruvate dehydrogenase, cytosolic malate dehydrogenase, lactate dehydrogenase, carnitine palmitoyltransferase 1 (CPT1) and CPT2, acyl CoA dehydrogenase, and UCP1 were analyzed by western blotting. In brief, homogenized BAT and WAT were solubilized in Laemmli buffer containing 2-mercaptoethanol. The proteins obtained were separated on denaturing SDS-PAGE 15\% (w/v) polyacrylamide gels. Equal amounts of proteins ( $20 \mu \mathrm{g} / \mathrm{lane})$, estimated using bicinchoninic acid reagent (Pierce, Rockford, IL, USA), were loaded into the gels. Proteins were blotted onto nitrocellulose (Immobilion-P; Millipore, Madrid, Spain) and the blots were blocked overnight at $4{ }^{\circ} \mathrm{C}$ with $5 \%$ (w/v) nonfat dry milk as reported (previously Modrego et al. 2013). The membranes were then incubated with different antibodies against each of the aforementioned proteins. For this purpose, 1,6-biphosphate aldolase and glyceraldehyde-3-phosphate dehydrogenase were determined using a polyclonal antibody and a MAB respectively (sc-12061 and sc-47724 respectively, Santa Cruz Biotechnology, Inc., 1:1000). Lactate dehydrogenase was determined using a MAB (sc-133123, Santa Cruz Biotechnology, Inc., 1:100). Pyruvate dehydrogenase and cytosolic malate dehydrogenase were determined by using a polyclonal antibody (sc-7140 and sc-49234, Santa Cruz Biotechnology, Inc., 1:1000). CPT1 and CPT2 were determined using polyclonal antibodies at a dilution of 1:1000 purchased from Santa Cruz Biotechnology, Inc. (sc-20670 and sc-20526 respectively). Acyl CoA dehydrogenase was determined by using a polyclonal antibody (ACAD-10 sc-1304 dilution 1:1000, Santa Cruz Biotechnology, Inc.). UCP1 was determined by using a polyclonal antibody (UCP1 sc-6529 dilution 1:1000, Santa Cruz Biotechnology, Inc.). The same samples were also incubated with a monoclonal anti- $\beta$ actin antibody as a loading control. After incubations, nitrocellulose membranes were washed and incubated with a peroxidase-conjugated anti-goat IgG antibody (for pyruvate dehydrogenase, cytosolic malate dehydrogenase, acyl CoA dehydrogenase, CPT2, and UCP1), a peroxidase-conjugated anti-rabbit IgG antibody (for CPT1), and with an anti-mouse IgG antibody (for glyceraldehydes-3-phosphate dehydrogenase, lactate dehydrogenase, and $\beta$-actin), and developed with ECL reagents (GE Healthcare, Little Chalfont Buckinghamshire, UK). Chemoluminiscence was evaluated by densitometry using Kodak Software (Kodak Gel Logic 2200, Imaging system densitometric Software). Prestained protein markers (Sigma) were used for molecular mass determinations.

\section{Determination of lactate content and, aconitase, lactate dehydrogenase, malate dehydrogenase activities}

Lactate content in adipose tissues and aconitase, lactate dehydrogenase, and malate dehydrogenase activities were determined by using the commercial colorimetric assay kits (BioVision Incorporated, Milpitas, CA, USA) following the manufacturer's instructions. To determine both lactate content and enzymatic activities, $80 \mu \mathrm{g}$ of each homogenized adipose tissue was used.

\section{Statistical analyses}

Statistical differences were analyzed using the MannWhitney's test. Results are expressed as mean \pm s.E.M. A $P$ value $<0.05$ was considered to be statistically significant. All data were analyzed using the SPSS Software Package (SPSS for Windows; SPSS, Inc., version 15.0).

\section{Results}

Table 2 lists the circulating metabolic characteristics of the rabbits. As was previously described, UCP1 was expressed in BAT but it was almost unexpressed in WAT, confirming the purity of the tissues used (Fig. 1).

Table 2 Lipid and glycolic circulating metabolic characteristics of the rabbits. Results represent the mean \pm s.E.M. of ten rabbits

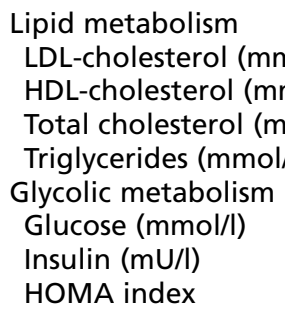

HOMA, homeostasis model assessment. 

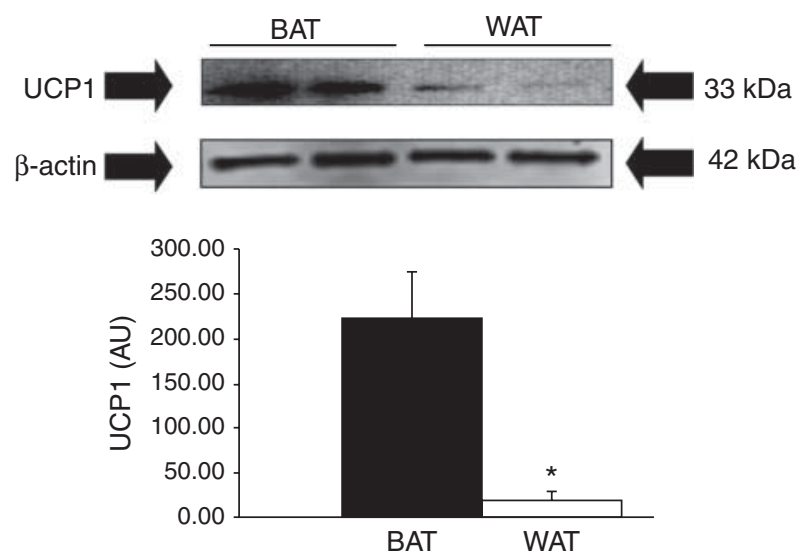

Figure 1

Representative western blot showing UCP1 expression in BAT and WAT. The expression of UCP1 protein was used to assess the purity of the tissues used. $\beta$-actin was used as a loading control. At the bottom, the bar graphs show the results of densitometric analysis of all the western blots in arbitrary units $(\mathrm{AU})$. The densitometric results represent the mean \pm s.E.M. of ten different rabbits. ${ }^{*} P<0.05$ with respect to BAT.

\section{Expression of proteins related to glucose and fatty acid metabolism in BAT and WAT}

Protein expression levels of fructose 1,6-biphosphate aldolase and glyceraldehyde-3-phosphate dehydrogenase, two glycolytic enzymes, were determined in BAT and WAT. The protein expression level of glyceraldehyde- 3-phosphate dehydrogenase was significantly higher in WAT than in BAT (Fig. 2). The expression level of 1,6-biphosphate aldolase also tended to be higher in WAT than in BAT, although it did not reach statistical significance (Fig. 2). Moreover, the expression level of pyruvate dehydrogenase, an enzyme that yields acetyl-CoA from pyruvate, was also significantly higher in WAT than in BAT (Fig. 2).

The protein expression level of the two fatty acid mitochondrial transporters, CPT1 and CPT2, was also determined in the both adipose tissues. BAT showed significantly higher expression levels of both CPT1 and CPT2 than WAT (Fig. 3). Moreover, the expression level of acyl CoA dehydrogenase, an enzyme involved in fatty acid $\beta$-oxidation, was also higher in BAT than in WAT (Fig. 3).

\section{Malate dehydrogenase, aconitase, and lactate complex}

Cytosolic malate dehydrogenase expression and activity were higher in WAT than in BAT (Fig. 4). There were no significant differences in aconitase activity between the two adipose tissues (Fig. 4).

There was markedly higher expression and activity of lactate dehydrogenase in BAT in comparison with WAT (Fig. 5). However, lactate dehydrogenase activity was similar in both adipose tissues (Fig. 5).
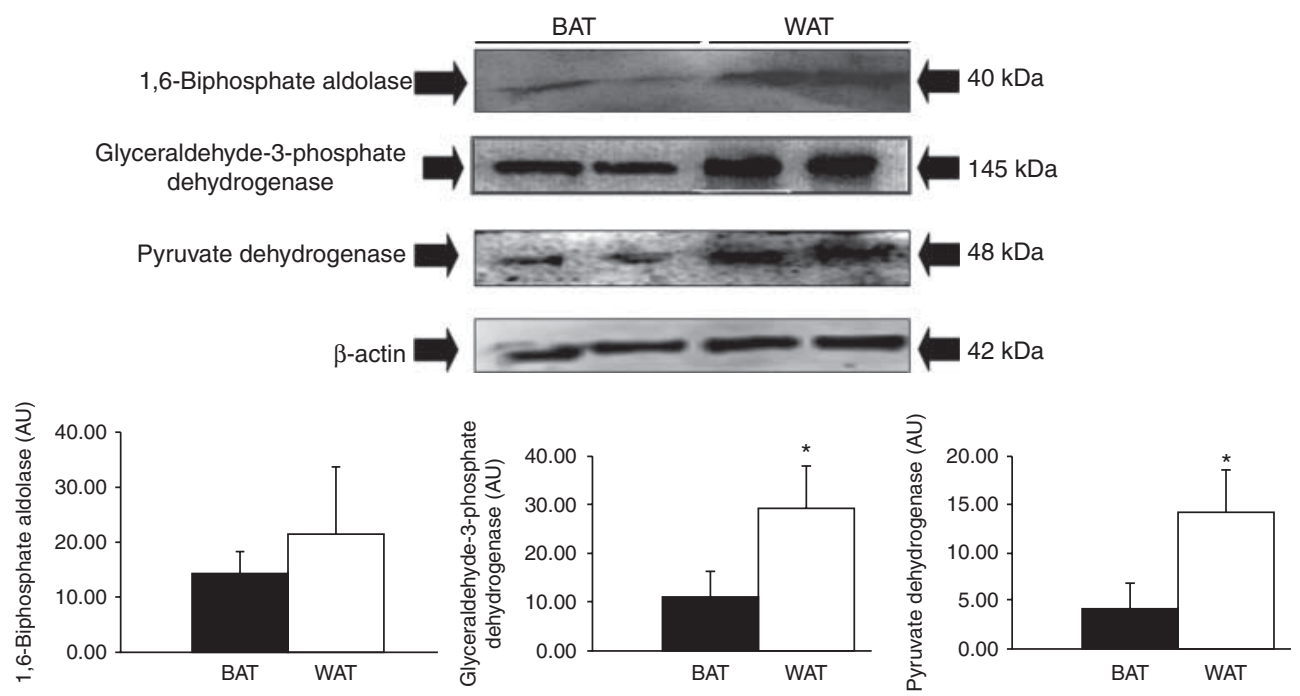

Figure 2

Representative western blots showing the expression of glycolytic enzymes in BAT and WAT obtained from rabbits. At the top, representative western blots showing the expression of 1,6-biphosphate aldolase, glyceraldehyde3-phosphate dehydrogenase, and pyruvate dehydrogenase in BAT and WAT. $\beta$-actin was used as a loading control. Each protein image corresponds to different gels with respect to the other proteins shown in the figure. At the bottom, the bar graphs show the results of densitometric analysis of all the western blots in arbitrary units. The densitometric results represent mean \pm S.E.M. of ten different rabbits. ${ }^{\star} P<0.05$ with respect to BAT. http://jme.endocrinology-journals.org DOI: $10.1530 / J M E-14-0255$
() 2015 Society for Endocrinology Printed in Great Britain
Published by Bioscientifica Ltd 

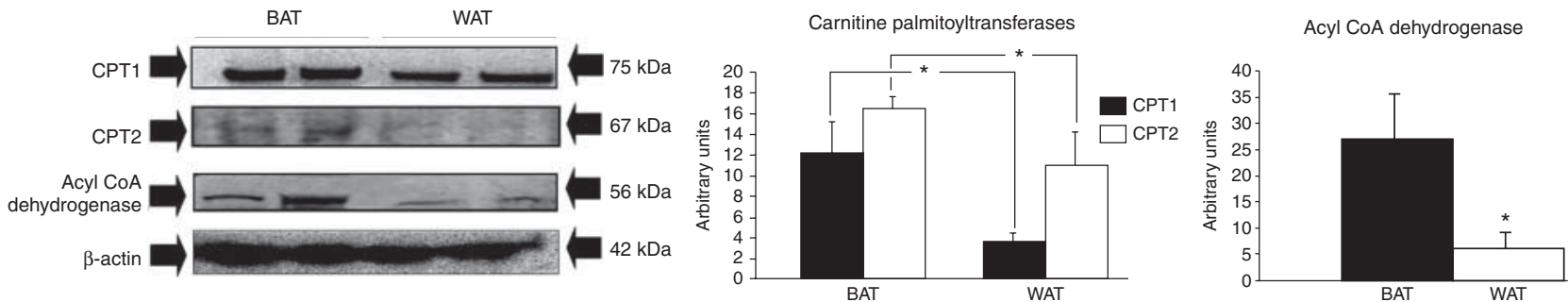

Figure 3

Representative western blots of the two fatty acid mitochondrial transporters, CPT1 and CPT2, and acyl CoA dehydrogenase, an enzyme involved in fatty acid $\beta$-oxidation, in BAT and WAT. At the left, a representative western blot showing the protein expression of fatty acid $\beta$-oxidation-related proteins. $\beta$-actin was used as a loading control.

\section{Discussion}

This study showed for the first time, to our knowledge, that, after 24-h fasting at physiological temperature, the expression and activities of proteins associated with gluconeogenesis are increased in WAT compared with BAT. However, BAT showed a greater expression of both of the
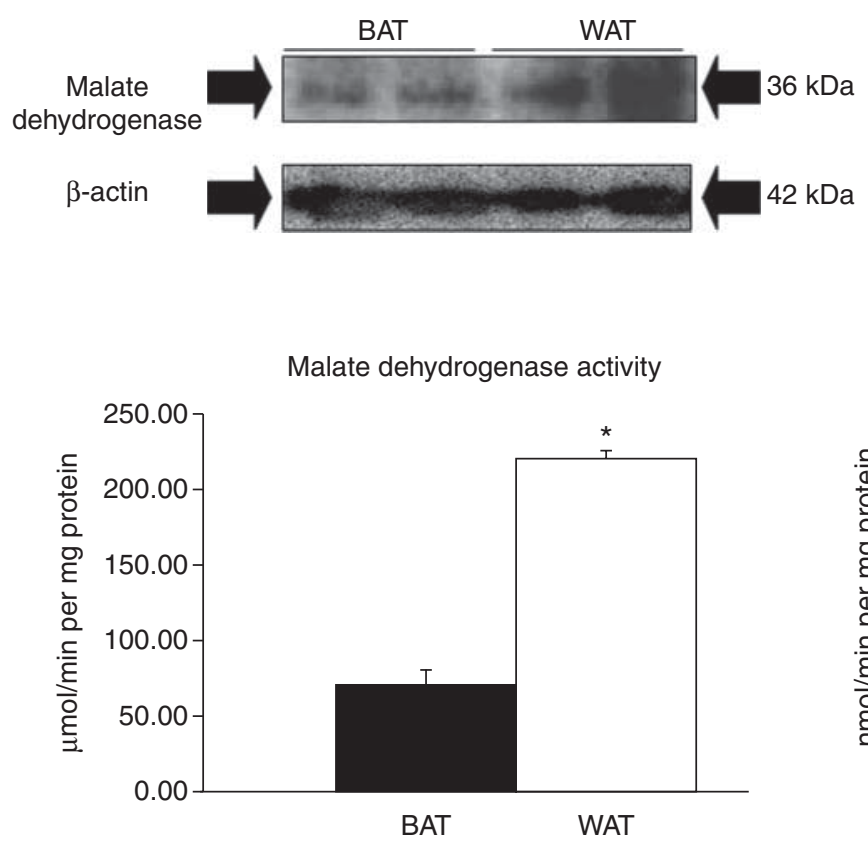

Figure 4

Protein expression of cytosolic malate dehydrogenase and enzymatic activities of aconitase and malate dehydrogenase in BAT and WAT. At the top left, there is a representative western blot of cytosolic malate dehydrogenase expression in BAT and WAT. $\beta$-actin was used as a loading control. At the top right the

$\begin{array}{lr}\text { http://jme.endocrinology-journals.org } & \text { C } 2015 \text { Society for Endocrinology } \\ \text { DOI: } 10.1530 / J M E-14-0255 & \text { Printed in Great Britain }\end{array}$

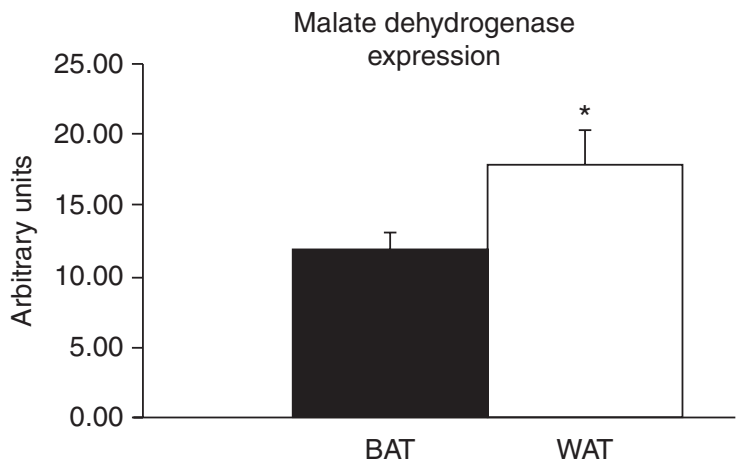

Each protein image corresponds to different gels with respect to the other proteins shown in the figure. At the middle and right, the bar graphs show the results of densitometric analysis of all the western blots in arbitrary units. The densitometric results are represent the mean \pm s.E.M. of ten different rabbits. ${ }^{\star} P<0.05$ with respect to BAT.

two main mitochondrial fatty acid transporters, CPT1 and CPT2, and also of an enzyme involved in fatty acid $\beta$-oxidation, acyl CoA dehydrogenase, than WAT. In addition, BAT showed higher lactate dehydrogenase expression and lactate content compared with WAT, indicating that under these experimental conditions the

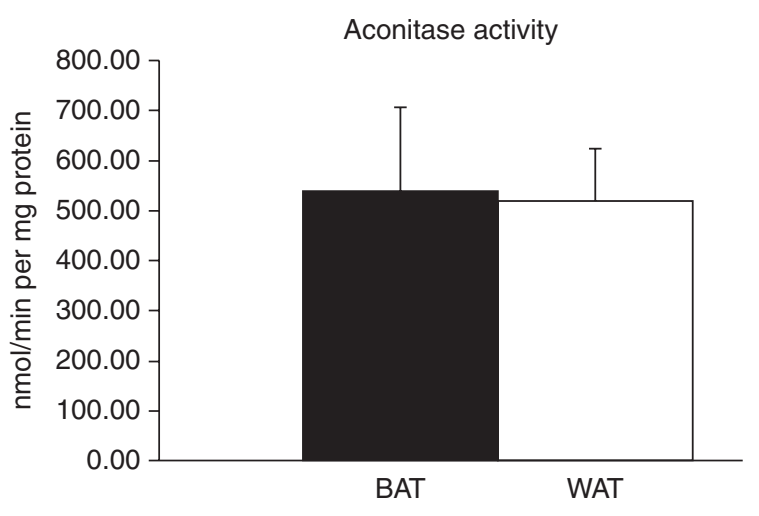

densitometric results of all the western blots in arbitrary units are shown. At the bottom, histograms show the enzymatic activities of aconitase and malate dehydrogenase in BAT and WAT. The results are represent the mean \pm s.E.M. of ten different rabbits. ${ }^{*} P<0.05$ with respect to BAT.

Published by Bioscientifica Ltd. 

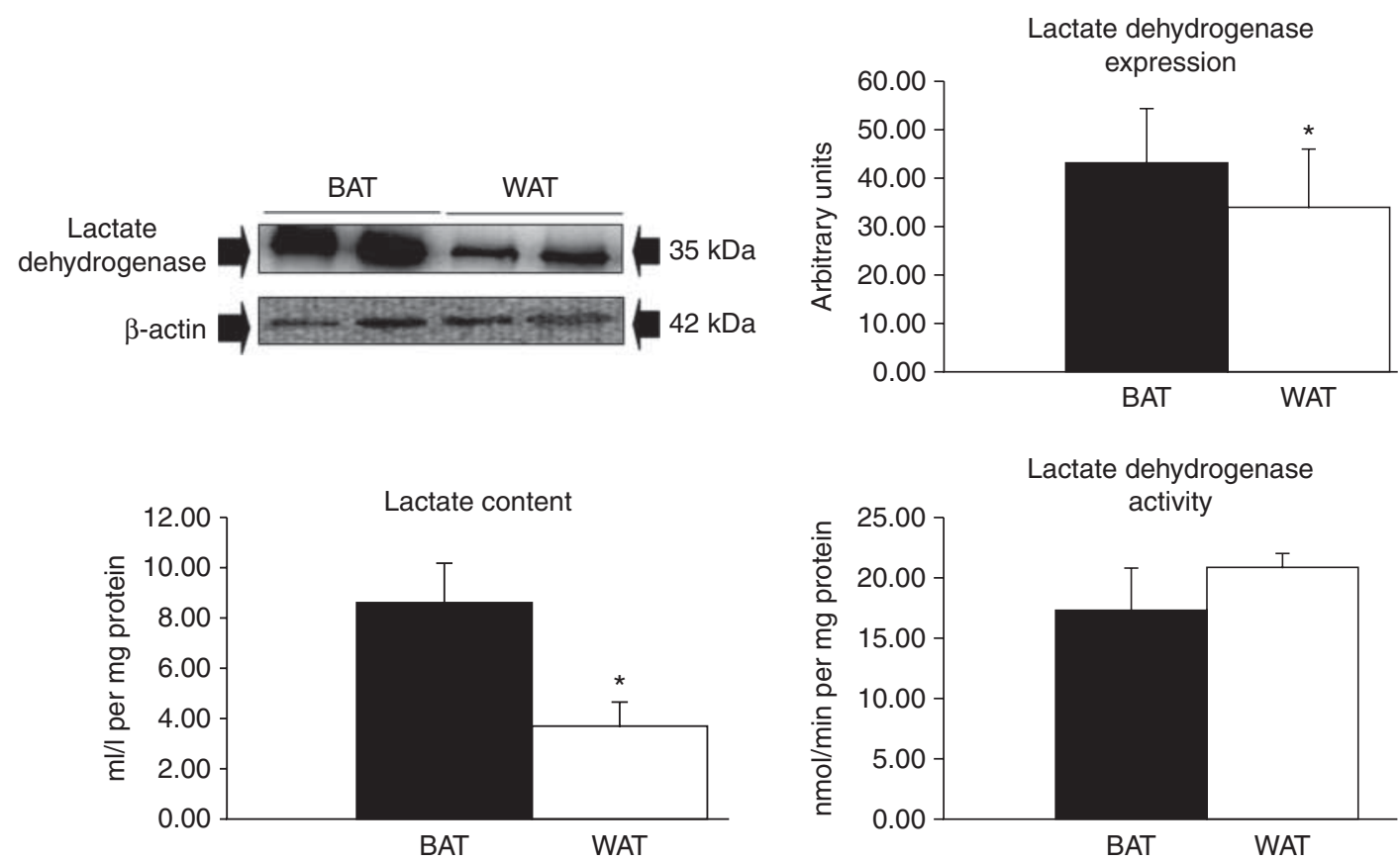

\section{Figure 5}

Lactate dehydrogenase expression, lactate content, and lactate dehydrogenase activity in BAT and WAT. At the top left is a representative western blot of lactate dehydrogenase expression in BAT and WAT. $\beta$-actin was used as a loading control. At the top right and bottom, histograms show the

anaerobic metabolism seems to be favored in BAT over WAT. In this regard, the increased content of lactate in BAT with respect to WAT seems to be dependent on increased expression of lactate dehydrogenase rather than changes in lactate dehydrogenase activity. In this regard, the fact that the expression of pyruvate dehydrogenase, one of the enzymes included in the pyruvate dehydrogenase complex that acts at the end of the glycolytic pathway controlling the conversion of pyruvate into acetyl-CoA, was significantly higher in WAT than in BAT may influence the increased expression of lactate dehydrogenase in BAT with respect to that found in WAT. Indeed, dehydrogenase deficiency has previously been associated with lactic acidosis (Brown et al. 1994).

Fasting has profound effects on glucose metabolism, resulting in a state of glucose intolerance. Glucose is also an essential component of adipose tissue metabolism (Cushman \& Wardzala 1980, Suzuki \& Kono 1980). In this study, after rabbits had fasted for $24 \mathrm{~h}$ at physiological temperature, WAT showed higher expression of one of the main enzymes involved in glycolysis, glyceraldehyde-3phosphate dehydrogenase, than that observed in BAT. Moreover, as mentioned above, pyruvate dehydrogenase expression was also significantly higher in WAT than in BAT. Taken together, these results may initially indicate that under lactate content and the enzymatic activity of lactate dehydrogenase in BAT and WAT. Results represent the mean \pm s.E.M. of ten different rabbits. $\star P<0.05$ with respect to BAT.

the conditions of physiological temperature and fasting, WAT may have higher rate of glucose catabolism than BAT. This could be subsequent to acceleration of lipolysis, because it is well known that lipolysis could be increased in WAT during fasting. However, results from previous studies have indicated that, under fasting conditions in rodents, WAT showed a reversible reduction in insulin-stimulated glucose transporters, decreasing the glucose oxidation rates. Interestingly, it was reported that binding of insulin to adipocytes from WAT was increased by fasting (Olefsky 1976). The discrepancy between the increased biding of insulin and the biological effect of the hormone has been suggested to be related to an acquired limitation of the cellular capacity for glucose metabolic activity (Olefsky 1976). Moreover, refeeding caused an overshoot in insulin-stimulated glucose uptake by WAT (Moore 1963).

Our results apparently also indicate that in WAT obtained from fasting rabbits at physiological temperature, the tricarboxylic acid cycle may be increased as compared with BAT. In this regard, although the activity of aconitase, an enzyme involved in the tricarboxylic acid cycle, was similar between WAT and BAT, an increased activity and expression of malate dehydrogenase was observed in WAT with respect to BAT. However, malate

Published by Bioscientifica Ltd 
dehydrogenase does not only participate in citrate formation in the mitochondria through the tricarboxylic acid cycle. In the adipose tissue, there is another cytosolic malate dehydrogenase isoform that reduces oxaloacetate to malate, releasing $\mathrm{NAD}+$. In the cytosol, malate dehydrogenase is part of the malate-aspartate shuttle involved in gluconeogenesis (Musrati et al. 1998). For this reason, in our experiments, a MAB that recognized the $\mathrm{N}$-terminal site of the cytosolic malate dehydrogenase was used. Taken together, these results support the hypothesis that the malate-aspartate shuttle may be increased in WAT with respect to in comparison with BAT.

The question then raised is why WAT has increased cytosolic malate dehydrogenase expression and activity after a 24-h fasting period at physiological temperature. Furthermore, to transport oxaloacetate outside the mitochondria, as oxaloacetate cannot leave the mitochondria, one of the main contributions of the malate-aspartate shuttle is the regeneration of NADH inside the mitochondrial matrix. This is potentially important because most of the gluconeogenic enzymes are located in the cytoplasm. In cytosol, malate is converted by the cytoplasmic malate dehydrogenase into oxaloacetate which is then converted into phosphoenolpyruvate to participate in glucose synthesis. The use of malate in the cytoplasm has advantages because the conversion of malate into oxaloacetate produces $\mathrm{NADH}$ that will be further required for the glyceraldehyde-3-phosphate dehydrogenase step during gluconeogenesis (Berg et al. 2002). Accordingly, as mentioned above, glyceraldehyde-3-phosophate dehydrogenase, an enzyme involved in both glycolysis and gluconeogenesis, has been found to be increased in WAT with respect to BAT. Moreover, the protein expression level of 1,6-biphosphate aldolase, an enzyme also involved in both glycolysis and gluconeogenesis, tended to be higher in WAT as compared with BAT, although the difference did not reach statistical significance.

Result from several studies have indicated that when glucose availability increases, fat oxidation decreases (Wolfe \& Peters 1987, Wolfe et al. 1988). Therefore, speculating with regard to these results as a whole, $24 \mathrm{~h}$ after starvation gluconeogenesis may be promoted in WAT as a counterbalancing mechanism against a putative increased triglyceride metabolism thus preventing total depletion of the lipid droplet in white adipocytes.

It has been previously reported that in rodents, BAT displays very active glycolysis, especially under sympathetic activation (Shimizu et al. 1991, 1993, Cypess et al. 2013). However, this hypothesis was mainly based on the fact that glucose uptake and the expression and translocation of specific glucose transporters were found to be increased in activated BAT (Shimizu et al. 1991, 1993, Cypess et al. 2013). In the present work, the expression levels of enzymes involved in glycolysis, i.e. glyceraldehyde-3-phosphate dehydrogenase and pyruvate dehydrogenase, were significantly lower in BAT as compared with WAT, indicating a lesser glycolytic capacity of BAT than of WAT under the conditions of physiological temperature and fasting.

Intracellular control of fatty acid oxidation begins at the level of mitochondrial uptake of fatty acid by the fatty acid transporters CPT1 and CPT2. These mitochondrial fatty acid transporters transfer fatty acid from acyl CoA to long-chain acyl-carnitine, which is then transported into mitochondria (Bonnefont et al. 2004). Under fasting conditions and at physiological environmental temperature, expression of both CPT1 and CPT2 proteins was markedly higher in BAT than in WAT. Moreover, one of the proteins involved in long-chain fatty acid $\beta$-oxidation, acyl CoA dehydrogenase also showed significantly higher levels of expression in BAT than in WAT. Taken together, these results may indicate that, under the conditions of $24-\mathrm{h}$ fasting at physiological temperature, fatty acid $\beta$-oxidation may be potentiated in BAT compared with WAT. It could be also in accordance with the observation that BAT has a higher mitochondrial content than WAT and also with the observation in rodents that fatty acids are physical activators of UCP1 (Marette \& Bukowiecki 1991). Moreover, active BAT is considered to be a major consumer of lipids and free fatty acids and its contribution to plasma clearance of administered triglycerides has been previously reported (Hagberg et al. 2010, Bartelt et al. 2012). On the other hand, an increased release of free fatty acids during thermogenesis from BAT has been assumed, indicating a role in substrate supply to other tissues (Nedergaard \& Lindberg 1979, Heldmaier \& Seidl 1985). However, the finding that the lactate content was increased in BAT compared with WAT diminished the importance of aerobic fatty acid $\beta$-oxidation as energetic fuel for BAT activity under the conditions of fasting and normal temperature. Therefore, although under the conditions of fasting and normal temperature BAT has apparently prepared the metabolic machinery for active fatty acid oxidation, BAT uses the anaerobic metabolism better than the aerobic metabolism to produce energy. However, the fact that different proteins involved in fatty acid oxidation appeared to be increased in BAT under the conditions of normal temperature and fasting may be reflecting the fact that BAT has been prepared to quickly use this metabolic pathway when it may be required, for example, under the

Published by Bioscientifica Ltd. 
conditions of cold-temperature or refeeding, through sympathetic and insulin-dependent mechanisms. Accordingly, as mentioned above, it is well established that sympathetic activation of BAT upregulates BAT fatty acid oxidation by increasing lipolysis, and by enhancing CPT activities and entry of fatty acids into the mitochondria (Nedergaard \& Lindberg 1979, Rothwell \& Stock 1983, Heldmaier \& Seidl 1985). Accordingly, it is a classical observation that the thermogenic process in brown adipocytes can be mimicked by the addition of fatty acids (Prusiner et al. 1968). Therefore, the activation of lipolysis is a sufficient trigger for initiation of thermogenesis in brown adipocytes. In this regard, it is also plausible that under the conditions of normal temperature and fasting, and to maintain adequate intracellular triglyceride storage, BAT uses lactate to form new fatty acids that may be essential during BAT activation. Indeed, it has been previously reported that in BAT lactate may be converted into fatty acids in fasted-refeed rats but not in fed or fasted animals (Schmidt \& Katz 1969).

In summary, this study described for the first time, to our knowledge, how that in rabbits under the conditions of physiological temperature and 24-h fasting, BAT seems to use anaerobic metabolism to provide energetic fuel, even though it has powerful fatty acid catabolic machinery. However, in WAT the malate-aspartate shuttle and therefore, the gluconeogenic pathway, seem to be potentiated under these experimental conditions. Better knowledge of the metabolic characteristics of BAT and WAT under different conditions improves the understanding of physiological processes and could be useful in the design of therapeutic interventions for metabolic disorders.

\section{Declaration of interest}

All authors have read and approved the submission of the manuscript and they have approved the final version of the manuscript. The material in the manuscript has not been published and is not being considered for publication elsewhere in whole or in part in any language. Moreover conflicts of interest are not involved.

\section{Funding}

This work was supported by Redes Temáticas de Investigación Cooperativa (RETICs) RD12/0042/0040 and RD12/0042/0033, Fondo Europeo de Desarrollo Regional (Fondos FEDER). J M is a staff member of RETICs. J J $Z-L$ is a staff member of the Comunidad de Madrid (S2010/BMD-2374). P R-S is a staff member of Red Heracles (Redes Temáticas de Cooperación Red Heracles (RETICS) RD06/0009/010).

\section{Acknowledgements}

The authors thank Begoña Larrea for secretarial assistance.

\section{References}

Athenstaedt K \& Daum G 2006 The life cycle of neutral lipids: synthesis, storage and degradation. Cellular and Molecular Life Sciences 63 1355-1369. (doi:10.1007/s00018-006-6016-8)

Bartelt A, Bruns OT, Reimer R, Hohenberg H, Ittrich H, Peldschus K, Kaul MG, Tromsdorf UI, Weller H, Waurisch C et al. 2011 Brown adipose tissue activity controls triglyceride clearance. Nature Medicine 17 200-205. (doi:10.1038/nm.2297)

Bartelt A, Merkel M \& Heeren J 2012 A new, powerful player in lipoprotein metabolism: brown adipose tissue. Journal of Molecular Medicine $\mathbf{9 0}$ 887-893. (doi:10.1007/s00109-012-0858-3)

Berg JM, Tymoczko JL \& Stryer L 2002 18.5.1 Many shuttles allow movement across the mitocondrial membranes. In Biochemistry, 5th edn. New York, NY, USA: W H Freeman.

Bonnefont JP, Djouadi F, Prip-Buus C, Gobin S, Munnich A \& Bastin J 2004 Carnitine palmitoyltransferases 1 and 2: biochemical, molecular and medical aspects. Molecular Aspects of Medicine 25 495-520. (doi:10.1016/ j.mam.2004.06.004)

Brown GK, Otero LJ, LeGris M \& Brown RM 1994 Pyruvate dehydrogenase deficiency. Journal of Medical Genetics 31 875-879. (doi:10.1136/jmg.31. 11.875)

Bukowiecki LJ 1989 Energy balance and diabetes: the effects of cold exposure, exercise training, and diet composition on glucose tolerance and glucose metabolism in rat peripheral tissues. Canadian Journal of Physiology and Pharmacology 67 382-393. (doi:10.1139/y89-062)

Cannon B \& Nedergaard J 2004 Brown adipose tissue: function and physiological significance. Physiological Reviews 84 277-359. (doi:10.1152/physrev.00015.2003)

Cushman SW \& Wardzala LJ 1980 Potential mechanism of insulin action on glucose transport in the isolated rat adipose cell. Apparent translocation of intracellular transport systems to the plasma membrane. Journal of Biological Chemistry 255 4758-4762.

Cypess AM, Doyle AN, Sass CA, Huang TL, Mowschenson PM, Rosen HN, Tseng YH, Palmer EL III \& Kolodny GM 2013 Quantification of human and rodent brown adipose tissue function using ${ }^{99 \mathrm{~m}} \mathrm{Tc}$-methoxyisobutylisonitrile SPECT/CT and ${ }^{18}$ F-FDG PET/CT. Journal of Nuclear Medicine 54 1896-1901. (doi:10.2967/jnumed.113.121012)

Dawkins MJ \& Hull D 1964 Brown adipose tissue and the response of new-born rabbits to cold. Journal of Physiology 172 2-38. (doi:10.1113/ jphysiol.1964.sp007414)

Festuccia WT, Blanchard PG, Turcotte V, Laplante M, Sariahmetoglu M, Brindley DN, Richard D \& Deshaies Y 2009 The PPAR $\gamma$ agonist rosiglitazone enhances rat brown adipose tissue lipogenesis from glucose without altering glucose uptake. American Journal of Physiology. Regulatory, Integrative and Comparative Physiology 296 R1327-R1351. (doi:10.1152/ajpregu.91012.2008)

Hagberg CE, Falkevall A, Wang X, Larsson E, Huusko J, Nilsson I, van Meeteren LA, Samen E, Lu L, Vanwildemeersch M et al. 2010 Vascular endothelial growth factor B controls endothelial fatty acid uptake. Nature 464 917-921. (doi:10.1038/nature08945)

Heldmaier G \& Seidl K 1985 Plasma free fatty acid levels during cold-induced and noradrenaline-induced nonshivering thermogenesis in the Djungarian hamster. Journal of Comparative Physiology. B, Biochemical, Systemic, and Environmental Physiology 155 679-684. (doi:10.1007/BF00694581)

Lean ME 1989 Brown adipose tissue in humans. Proceedings of the Nutrition Society 48 243-256. (doi:10.1079/PNS19890036)

Lin CS \& Klingenberg M 1980 Isolation of the uncoupling protein from brown adipose tissue mitochondria. FEBS Letters 113 299-303. (doi:10.1016/0014-5793(80)80613-2)

Marette A \& Bukowiecki LJ 1991 Noradrenaline stimulates glucose transport in rat brown adipocytes by activating thermogenesis: evidence that fatty acid activation of mitochondrial respiration enhances glucose transport. Biochemical Journal 277 119-124.

Published by Bioscientifica Ltd. 
Martin ML \& Jensen MD 1991 Effects of body fat distribution on regional lipolysis in obesity. Journal of Clinical Investigation 88 609-613. (doi:10. 1172/JCI115345)

Modrego J, de las Heras N, Zamorano-León JJ, Mateos-Cáceres PJ Martín-Fernández B, Valero-Muñoz M, Lahera V \& López-Farré AJ 2013 Changes in cardiac energy metabolic pathways in overweight rats fed a high-fat diet. European Journal of Nutrition 52 847-856. (doi:10.1007/ s00394-012-0392-7)

Moore RO 1963 Influence of fasting and refeeding on response of adipose tissue to insulin. American Journal of Physiology 205 222-224.

Musrati RA, Kollárová M, Mernik N \& Mikulásová D 1998 Malate dehydrogenase: distribution, function and properties. General Physiology and Biophysics 17 193-210.

Nedergaard J \& Lindberg O 1979 Norepinephrine-stimulated fatty-acid release and oxygen consumption in isolated hamster brown-fat cells. Influence of buffers, albumin, insulin and mitochondrial inhibitors. European Journal of Biochemistry 95 139-145. (doi:10.1111/j.1432-1033. 1979.tb12948.x)

Olefsky JM 1976 Effects of fasting on insulin binding, glucose transport, and glucose oxidation in isolated rat adipocytes: relationships between insulin receptors and insulin action. Journal of Clinical Investigation $\mathbf{5 8}$ 1450-1460. (doi:10.1172/JCI108601)

Prusiner SB, Cannon B \& Lindberg O 1968 Oxidative metabolism in cells isolated from brown adipose tissue. I. Catecholamine and fatty acid stimulation of respiration. European Journal of Biochemistry 6 15-22. (doi:10.1111/j.1432-1033.1968.tb00413.x)
Rothwell NJ \& Stock MJ 1983 Effects of age on diet-induced thermogenesis and brown adipose tissue metabolism in the rat. International Journal of Obesity 7 583-589.

Schmidt K \& Katz J 1969 Metabolism of pyruvate and L-lactate by rat adipose tissue. Journal of Biological Chemistry 44 2125-2131.

Shimizu Y, Nikami H \& Saito M 1991 Sympathetic activation of glucose utilization in brown adipose tissue in rats. Journal of Biochemistry 110 688-692.

Shimizu Y, Nikami H, Tsukazaki K, Machado UF, Yano H, Seino Y \& Saito M 1993 Increased expression of glucose transporter GLUT-4 in brown adipose tissue of fasted rats after cold exposure. American Journal of Physiology 264 E890-E895.

Suzuki K \& Kono T 1980 Evidence that insulin causes translocation of glucose transport activity to the plasma membrane from an intracellular storage site. PNAS 77 2542-2545. (doi:10.1073/ pnas.77.5.2542)

Szepesi B \& Berdanier CD 1971 Time course of the starve-refeed response in rats; possible role of insulin. Journal of Nutrition 101 1563-1574.

Trayhurn P \& Beattie JH 2001 Physiological role of adipose tissue: white adipose tissue as an endocrine and secretory organ. Proceedings of the Nutrition Society 60 329-339. (doi:10.1079/PNS200194)

Wolfe RR \& Peters EJ 1987 Lipolytic response to glucose infusion in human subjects. American Journal of Physiology 252 E218-E223.

Wolfe BM, Klein S, Peters EJ, Schmidt BF \& Wolfe RR 1988 Effect of elevated free fatty acids on glucose oxidation in normal humans. Metabolism $\mathbf{3 7}$ 323-329. (doi:10.1016/0026-0495(88)90131-X)

Received in final form 22 December 2014

Accepted 7 January 2015
(C) 2015 Society for Endocrinology Printed in Great Britain 